International Journal of Wireless \& Mobile Networks (IJWMN) Vol. 4, No. 6, December 2012

\title{
EFFECT OF ANTENNA SPACING ON THE Performance of Multiple INPUt Multiple OUTPUT LTE DOWNLINK IN AN URBAN MICROCELL
}

\author{
Sunil Joshi ${ }^{1}$, Deepak Gupta ${ }^{2}$, S. Z. Haque ${ }^{3}$, Neha Kothari ${ }^{1}$,P.C. Bapna ${ }^{1}$ \\ ${ }^{1}$ Department of Electronics \& Communication, CTE, MPUAT, Udaipur, India, \\ suniljoshi7@rediffmail.com \\ ${ }^{2}$ Department of Electronics, V.B. Polytechnic College, Udaipur, INDIA, \\ simptech2012@yahoo.in \\ ${ }^{3}$ Department of Electronics \& Information Technology, MCIT, Govt. of India, New \\ Delhi \\ haque@mit.gov.in
}

\begin{abstract}
The paper presents design of a $2 \times 2$ multiple input multiple output (MIMO) LTE Downlink using OFDM with 16-QAM scheme, operated in a spatial Multiplexing (SM) mode. An urban Microcell Winner channel model is assumed to investigate the performance of the system. The focus of this paper is to understand the effect of antenna spacing of end transceivers on the performance of $2 \times 2$ MIMO LTE Downlink. The performance parameters like Capacity, Throughput and Bit error rate are determined for different antenna spacing at Base station (BS) as well as at mobile station( $M S)$ for single user. Further the quantitative superiority of closed loop MIMO over Open Loop MIMO is established and discussed. The results depicted in the paper could be of vital importance for commercial deployment of MIMO based systems to fulfill requirements of contemporary wireless baseband technology.
\end{abstract}

\section{KEYWORDS}

Spatial Multiplexing, Spatial Diversity, Multiple Input- Multiple Output (MIMO), Space Time Coding, Rician factor.

\section{INTRODUCTION}

It is theoretically established fact that MIMO may be a potential technique supporting relatively high and robust data rates with increased spectral efficiency, and data throughput as compared to SISO system of identical bandwidth and transmitted power [1].Because of these attributes, it is being adopted for next generation wireless broadband systems [2]. MIMO transmission strategies can be broadly classified into diversity and spatial multiplexing. While diversity aims to lower the probability of error and thereby improve the reliability of the communication link, spatial multiplexing is used to increase the achievable data rates [3, 4] MIMO transmission strategies can also be classified as open-loop and closed-loop. In open-loop strategies, the transmitter does not have any knowledge of the channel and transmission follows a deterministic pattern that is independent of the channel. Open-loop transmission strategies include spatial multiplexing and diversity schemes like space time block codes, cyclic delay diversity etc. Closed loop schemes require knowledge of the channel at the transmitter to adapt the transmitted signal to the channel conditions, here the mobile station (MS) feeds back the CSI (channel state information) to Base Station (BS) for best utilization of MIMO Channel [5]. A detailed comparative study between closed loop and open loop MIMO schemes for OFDM based mobile broadband radio access for 3GPP UTR LTE is carried out to establish superiority of Closed loop MIMO over open loop MIMO [6] . 3GPP LTE is the evolution of the Thirdgeneration of mobile communications, UMTS, to the Fourth generation technology, which is DOI : 10.5121/ijwmn.2012.4613 
essentially a wireless broadband Internet system with voice and other services built on top. LTE is designed to increase data rates and cell edge bitrates, improve spectrum efficiency (unicast as well as broadcast) and allow spectrum flexibility $(1.25,2.5,5,10,15$ and $20 \mathrm{MHz}$ ) for flexible radio planning. [7]

In the literature, theoretical studies have been reported showing the effects of antenna spacing at the transmitter and the receiver sides, where the correlation coefficient of the incoming signals with respect to antenna spacing was investigated. The impact of antenna spacing on channel capacity has been measured intensively in a variety of scenarios and conditions [8,9]. Recently, the influence of antenna spacing on the throughput of an OFDM transmission was studied in $[10,11]$ by using sounded channel coefficients in a simulation. By far very few literature is available reporting the effect of open loop and close loop MIMO along with antenna spacing [13-18].

The performance analysis is performed in the downlink of a 3GPP LTE OFDMA system. Multiple input multiple output (MIMO) technologies introduced in LTE such as spatial multiplexing, transmit diversity, and beam forming are key components for providing higher peak rate at a better system efficiency, which are essential for supporting future broadband data service over wireless link [11]. LTE uses OFDMA on the downlink, which is well suited to achieve high peak data rates in high-spectrum bandwidth.

To evaluate the performance of MIMO strategies in LTE, different antenna spacing combinations have been considered. We have investigated the performance of the MIMO base station Antenna Spacing with reference to capacity, throughput and BLER of the System. Both open-loop and closed-loop Single User MIMO systems are discussed with particular emphasis on the data rate maximization aspect of MIMO.

\section{System Design And Parameters}

System Description: Figure 1 shows a simplified system block diagram of a 2X2 MIMO System

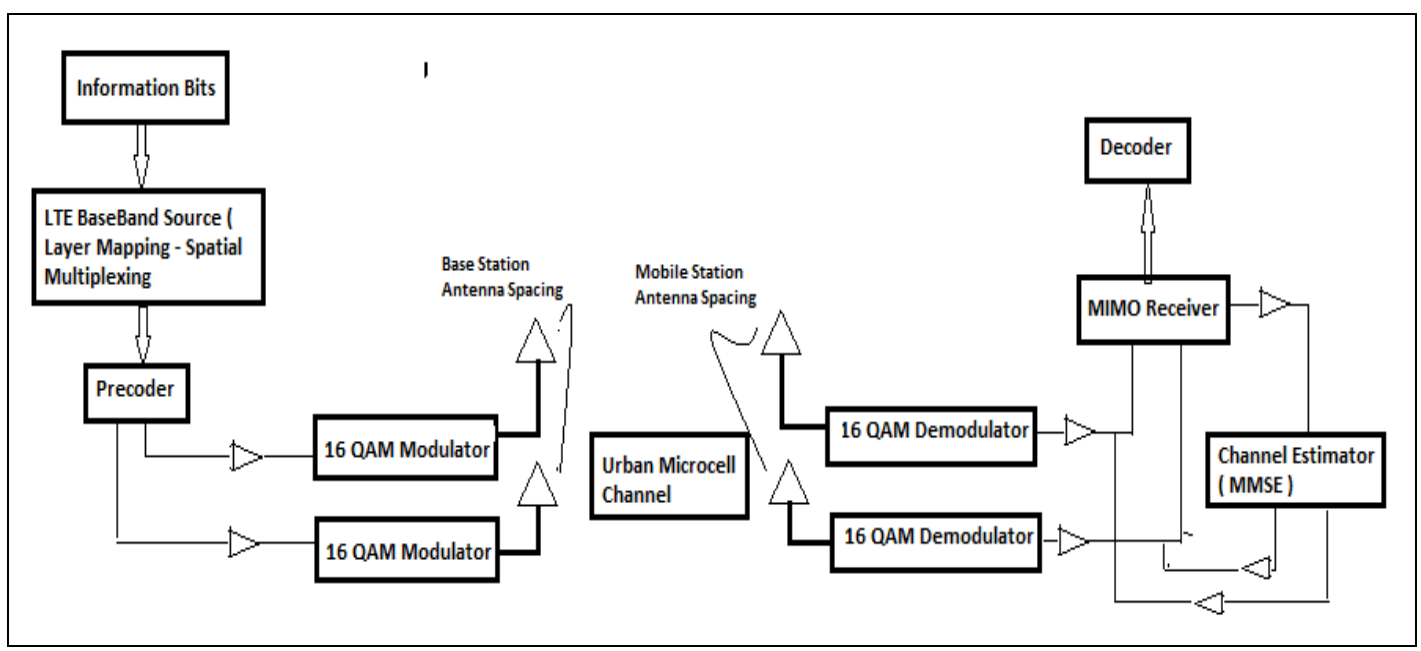

Figure $1.2 \times 2$ MIMO System

Figure 2 depicts a typical urban microcell propagation scenario is modeled as high rise buildings and down town streets with base station antenna height of typical lamp post height, much lower than the surrounding buildings and structures. The mobile station height is assumed to be 1.5 meters. The resultant signal at the receiver is the vector sum of multiple rays reaching through multiple reflections of the building walls. The main street and perpendicular streets length are kept at 500 meter each and the speed of mobile is fixed at $20 \mathrm{~km} / \mathrm{hr}$. 
International Journal of Wireless \& Mobile Networks (IJWMN) Vol. 4, No. 6, December 2012

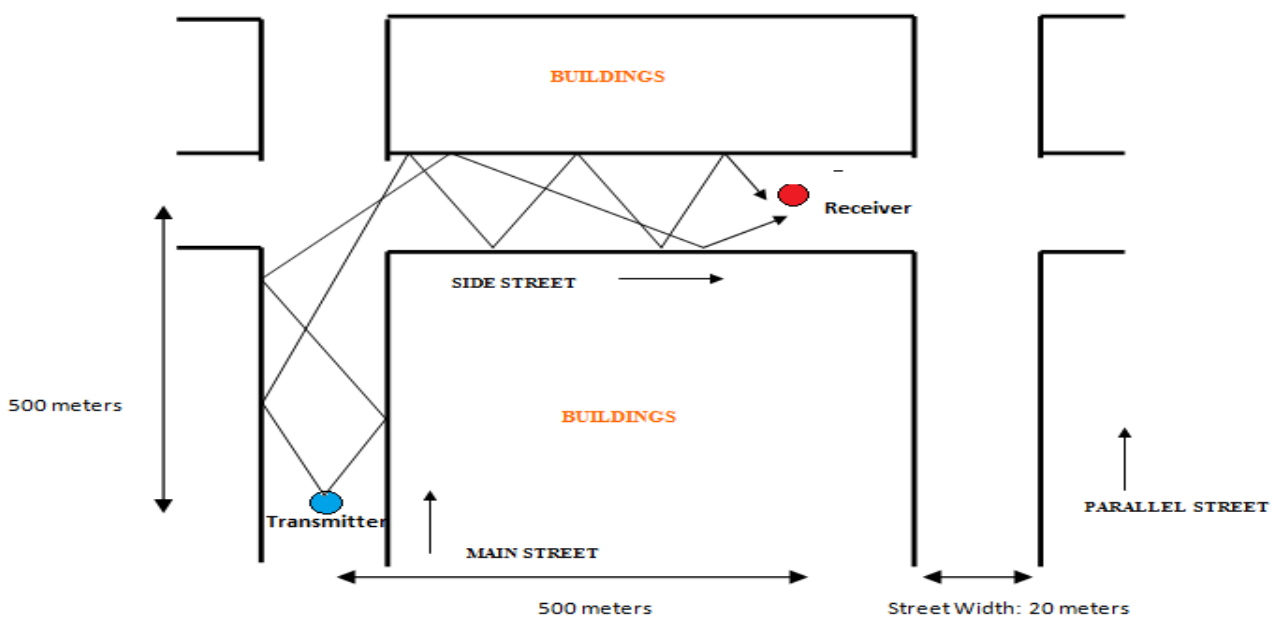

Figure 2. Geometry of Urban Microcell Scenario (NLOS)

The aim is to optimize antenna spacing for downlink in urban microcellular scenario. The system parameters are tabulated below in table 1 .

\begin{tabular}{|c|c|}
\hline Parameter & Specification \\
\hline Mode & $\begin{array}{l}\text { MIMO Open Loop Spatial Multiplexing } \\
\text { MIMO Closed Loop Spatial Multiplexing }\end{array}$ \\
\hline Main Street & 500 meters \\
\hline Perpendicular Street & 500 meters \\
\hline Transmitting Antenna & 3- Sector \\
\hline Receiving Antenna & Omni directional \\
\hline Frequency & $2.15 \mathrm{GHz}$ \\
\hline Modulation Scheme & 16QAM ( $1 / 2$ Code rate) \\
\hline Detection scheme & MMSE \\
\hline Vehicular Speed & $20 \mathrm{Km} / \mathrm{hr}$ \\
\hline Channel Bandwidth & $10 \mathrm{MHz}$ \\
\hline Transmitter height & 10 meters \\
\hline Receiver height & 1.5 meters \\
\hline Transmit Power & $10 \mathrm{dBm}$ \\
\hline
\end{tabular}

Table 1: System Parameters

\section{ObSERVATION}

(a) Capacity v/s SNR: 
International Journal of Wireless \& Mobile Networks (IJWMN) Vol. 4, No. 6, December 2012

The Capacity curves are as shown in figure 3 \& figure 4 respectively. The capacity analysis is undertaken to optimize antenna spacing at Base Station for downlink in urban microcell Scenario

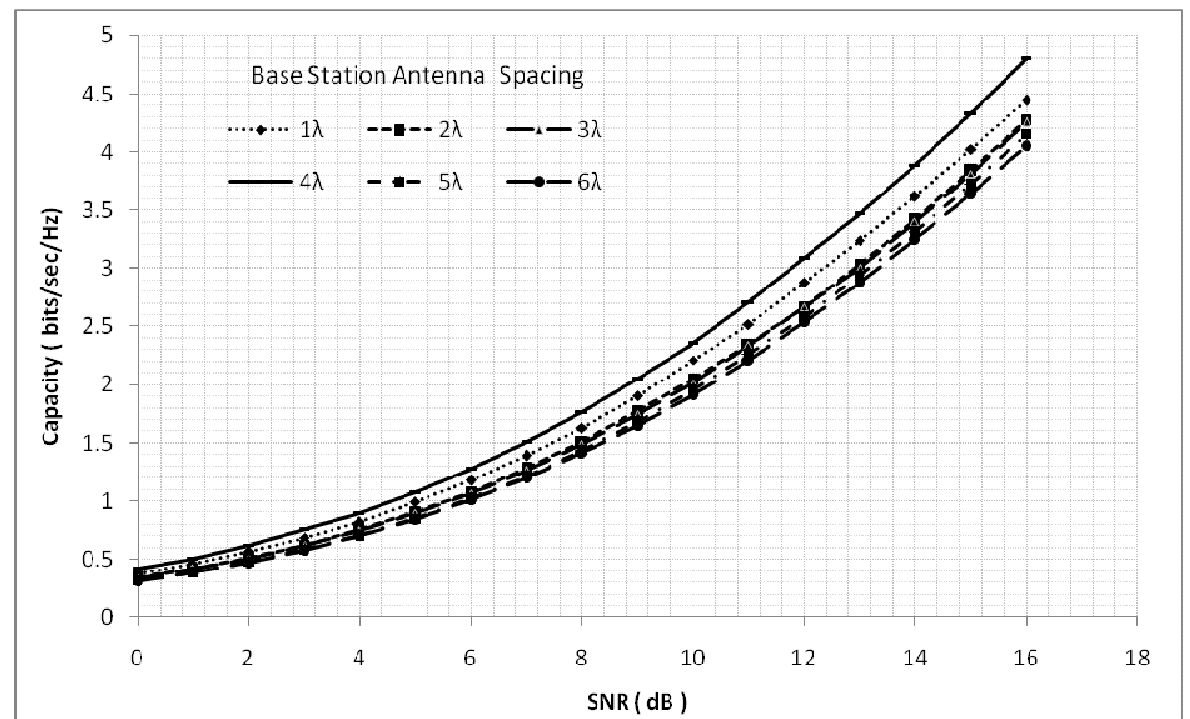

Figure 3. Capacity v/s SNR Plot for Open-Loop MIMO

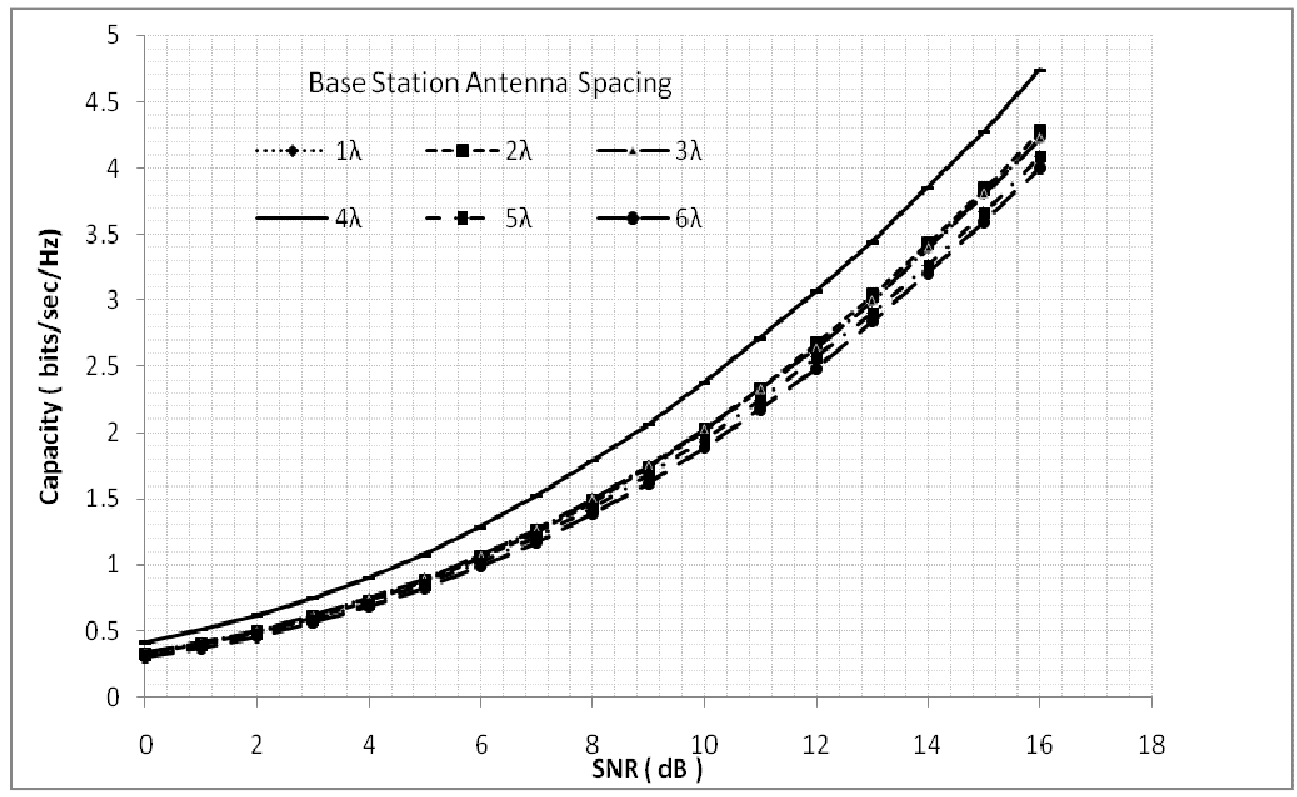

Figure 4. Capacity v/s SNR Plot for 2x2 Close-Loop MIMO

Referring to figure 3 and 4 the capacity increase in antenna spacing at Base station from $\lambda$ to $6 \lambda$ a small increase is observed, being maximum for an antenna spacing of $4 \lambda$ at all ranges of SNR. However for relatively lower SNR $(<=5 \mathrm{~dB})$ the capacity gain with varying antenna spacing is not found to be significant.. For moderate SNR ( $<=10 \mathrm{~dB}$ and $>=5) \mathrm{dB}$, the spacing effect is more pronounced in terms of capacity curves, it indicates increase in capacity almost linear to increase in SNR for fixed antenna spacing. 
International Journal of Wireless \& Mobile Networks (IJWMN) Vol. 4, No. 6, December 2012

The CDF of capacity curves for open loop and closed loop MIMO are shown in figures 5 and 6 respectively.

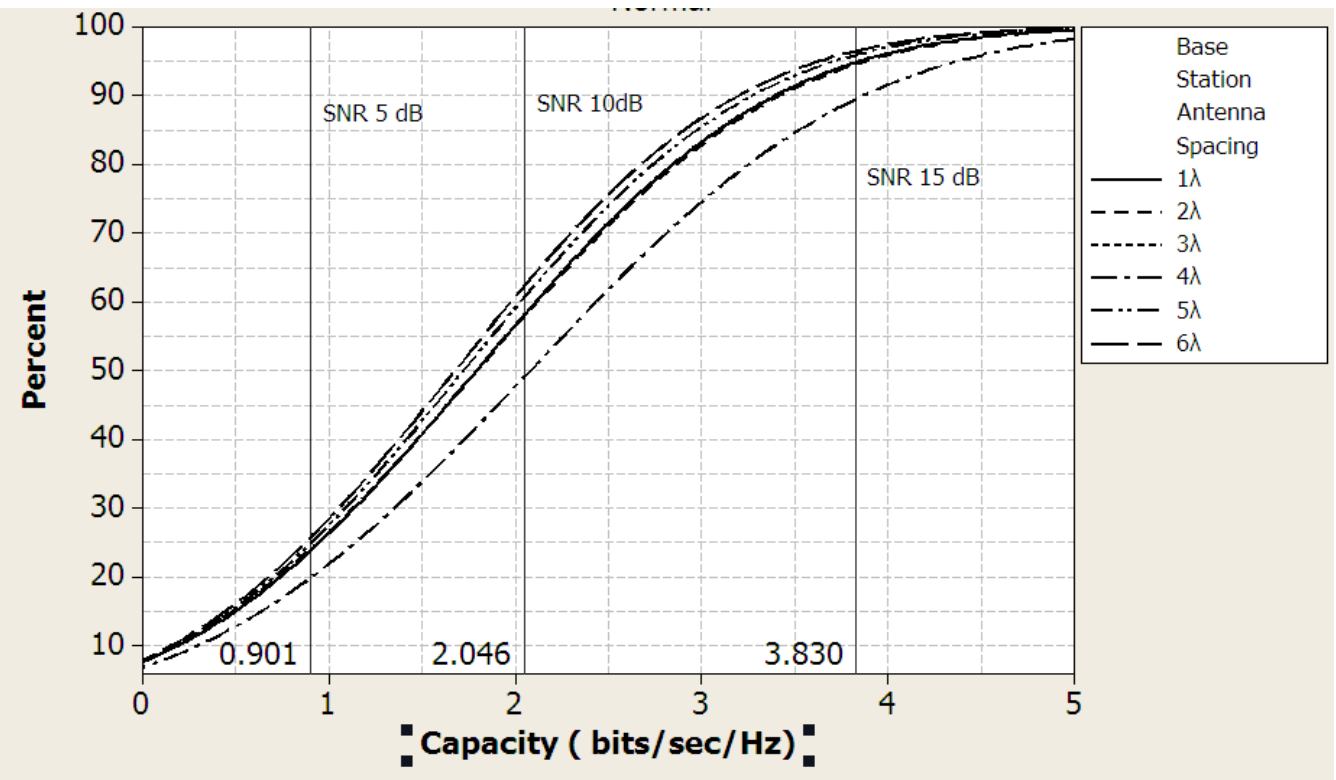

Figure 5. CDF Plot for capacity of Open-Loop MIMO

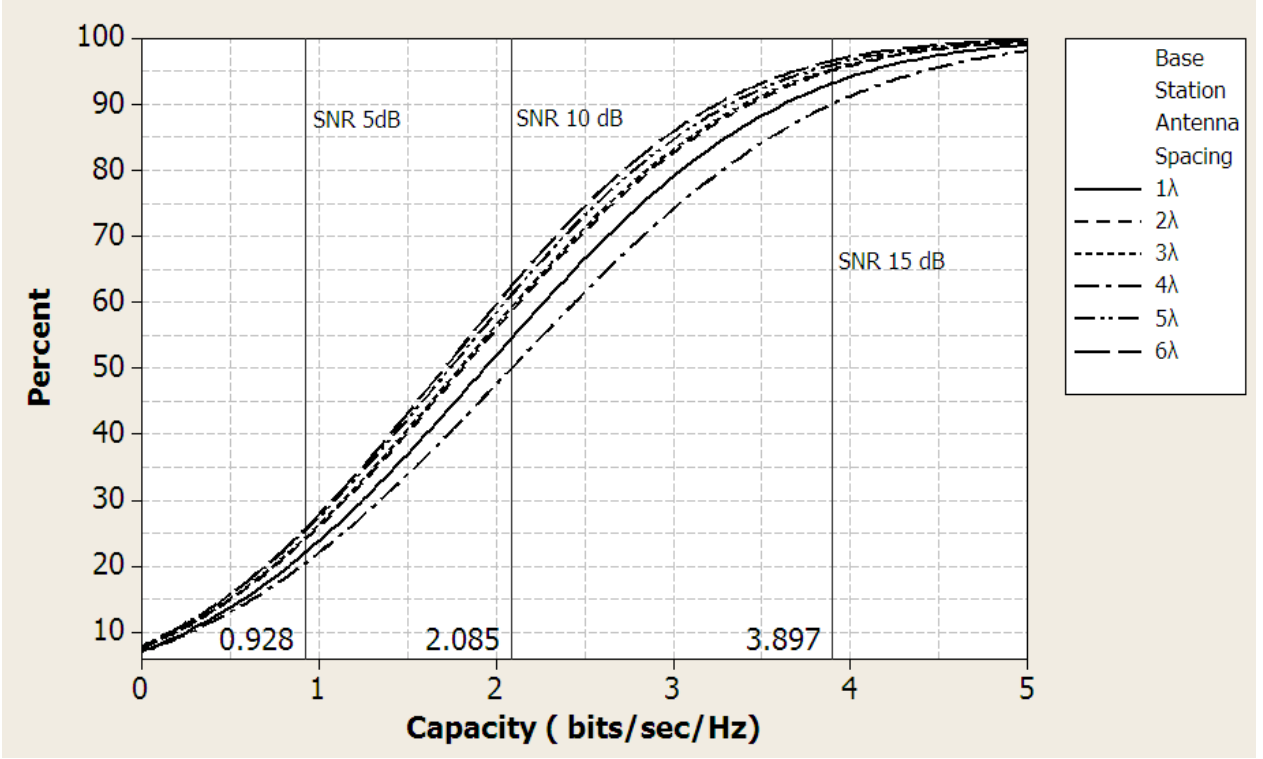

Figure 6. CDF Plot for capacity of Close -Loop MIMO

It can be observed that for relatively lower SNR $(<=5 \mathrm{~dB})$ close loop MIMO provides a marginal improvement of about $3 \%(0.027 \mathrm{bits} / \mathrm{sec} / \mathrm{Hz})$ over open loop MIMO with SNR gain of around $2 \mathrm{~dB}$.

The improvement for moderate SNR range ( $>5 \&<10$ ) is of around $1.9 \%(.049 \mathrm{bits} / \mathrm{sec} / \mathrm{Hz})$ and higher SNR values $(>10 \mathrm{~dB})$ is $1.7 \%(0.057 \mathrm{bits} / \mathrm{sec} / \mathrm{Hz})$.

\section{(b) Throughput v/s SNR:}


International Journal of Wireless \& Mobile Networks (IJWMN) Vol. 4, No. 6, December 2012

The throughput curves are as shown in figure 7 and 8 for Open and Close Loop MIMO respectively. The analysis is undertaken to optimize antenna spacing at Base Station for downlink in urban microcell Scenario.

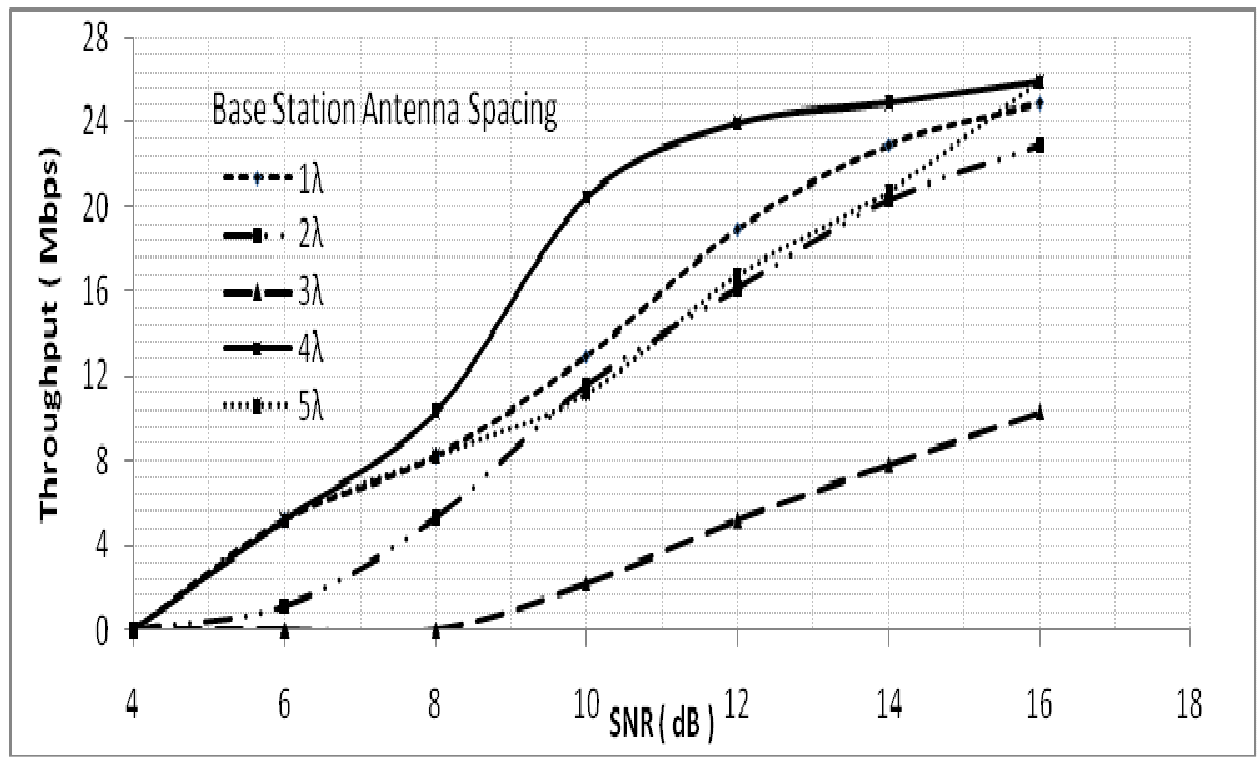

Figure 7. Throughput v/s SNR Plot for Open-Loop MIMO

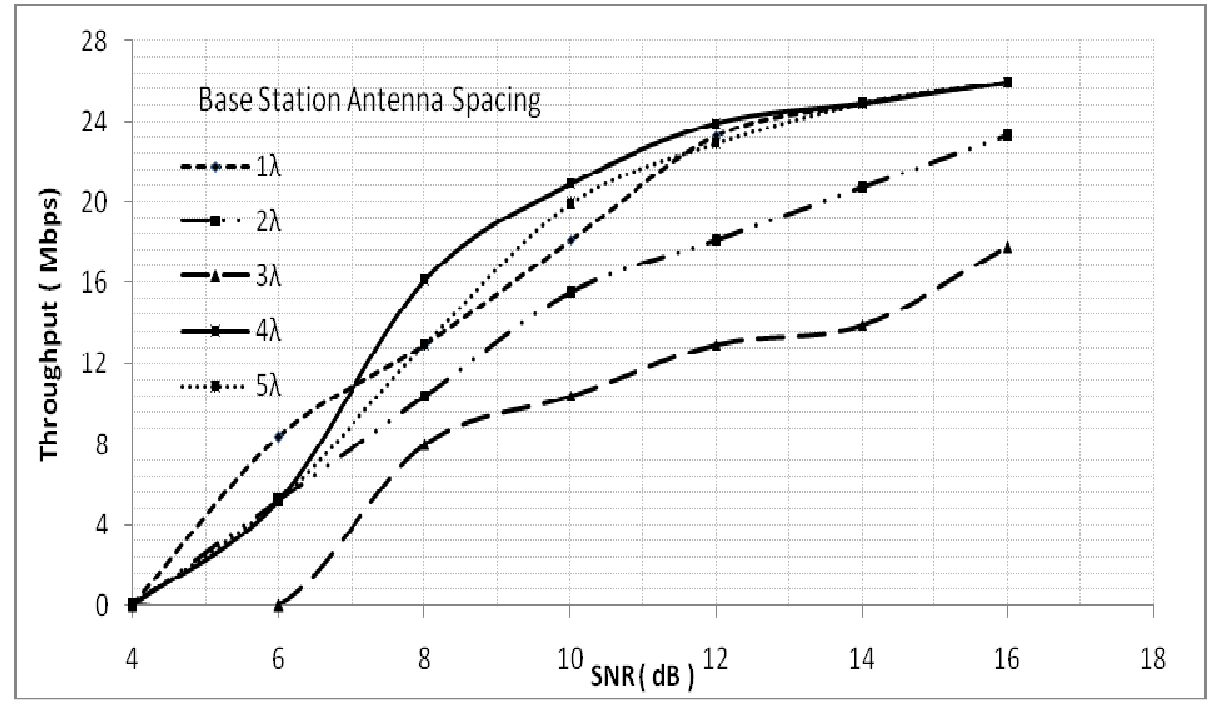

Figure 8. Throughput v/s SNR Plot for close-Loop MIMO

For relatively lower SNR ( $<=5 \mathrm{~dB}$ ) the antenna spacing $4 \lambda$ and $1 \lambda$ reveals identical throughput of $2 \mathrm{Mbps}$, however for other antenna spacing it is insignificant. The antenna spacing effect is pronounced at SNR $>=8 \mathrm{~dB}$. Antenna Spacing of $4 \lambda$ gives maximum throughput and $3 \lambda$ gives minimum throughput. One critical observation is that, throughput deteriorates with increase in base station antenna spacing till $3 \lambda$, however it starts improving beyond $3 \lambda$ for all ranges of SNR. As the channel conditions improves $(>=8 \mathrm{~dB}$ ) Increase in spacing from $1 \lambda$ to $6 \lambda$ has distinct effect on throughput, the largest being from $3 \lambda$ to $4 \lambda$. For Open Loop MIMO $4 \lambda$ 
International Journal of Wireless \& Mobile Networks (IJWMN) Vol. 4, No. 6, December 2012

achieves roughly $2 \mathrm{~dB}$ theoretical gain over $1 \lambda$ and $3 \mathrm{~dB}$ gain over $2 \lambda$ whereas, in close loop $1.2 \mathrm{~dB}$ gain is achieved over $1 \lambda$ and $2 \mathrm{~dB}$ gain over $2 \lambda$ is obtained at a reference throughput of 15 Mbps.

The CDF of throughput curves for open loop and closed loop MIMO are shown in figures 9 and 10 respectively.

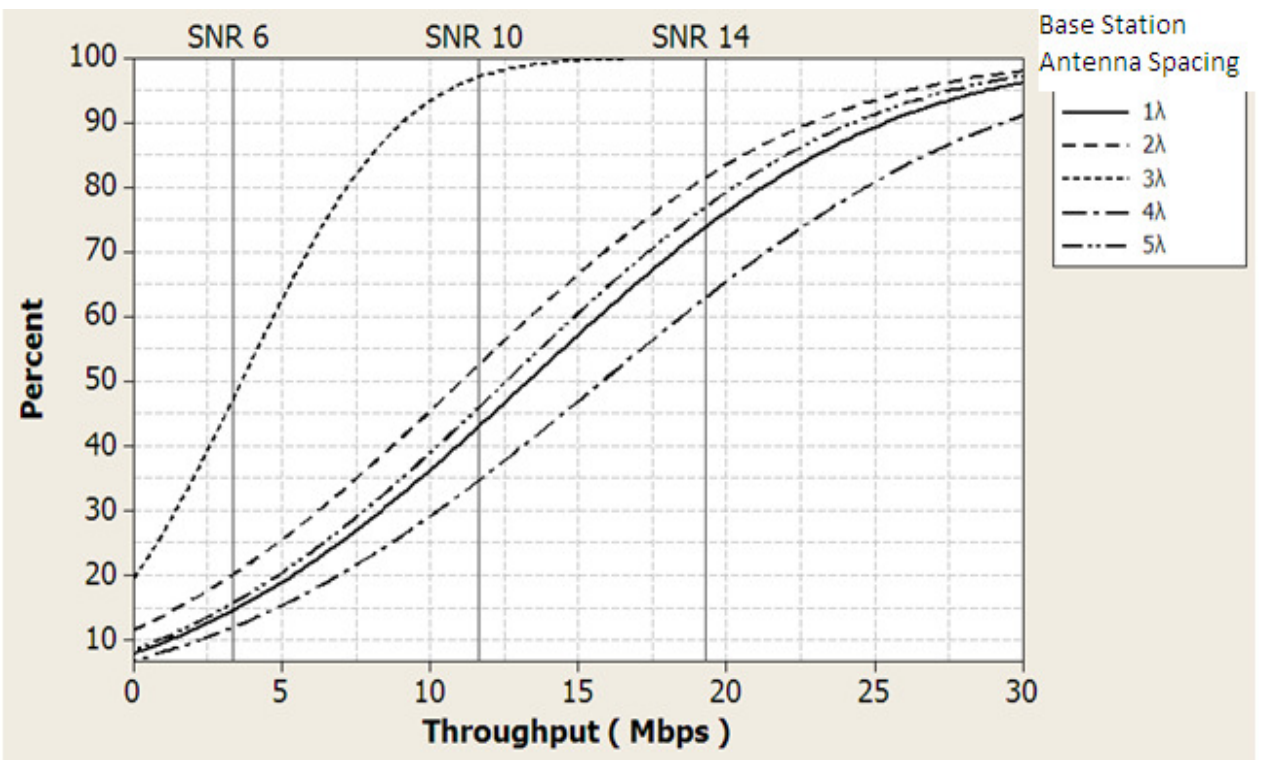

Figure 9. CDF Plot for Open-Loop MIMO

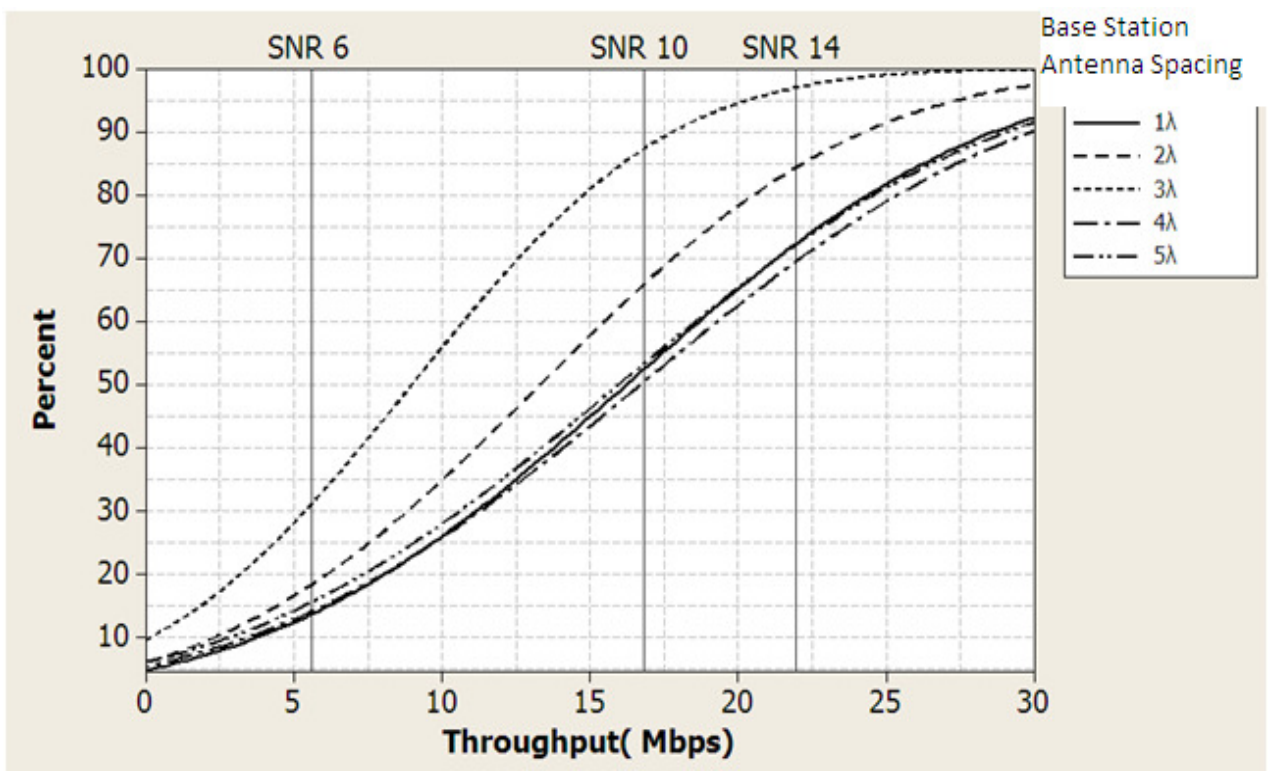

Figure 10. CDF Plot for Close -Loop MIMO 
International Journal of Wireless \& Mobile Networks (IJWMN) Vol. 4, No. 6, December 2012

It is observed that performance degrades in open loop MIMO compared to close loop MIMO for all SNR range. The throughput effect becomes more significant for higher $(>10 \mathrm{~dB})$ and moderate SNR ( 5 to $10 \mathrm{~dB}$ ) values. For moderate SNR values improvement from open loop to close loop is around 21\% (11.66 Mbps v/s $15.86 \mathrm{Mbps}$ ) whereas for High SNR range it reaches to $45 \%$ (18.66 Mbps v/s $22.96 \mathrm{Mbps}$ ) for all antenna spacing.

\section{(c) BLER V/S SNR:}

The BLER v/s SNR curves are as shown in figure 11 and 12. The analysis is undertaken to optimize antenna spacing at Base Station for downlink in urban microcell Scenario.

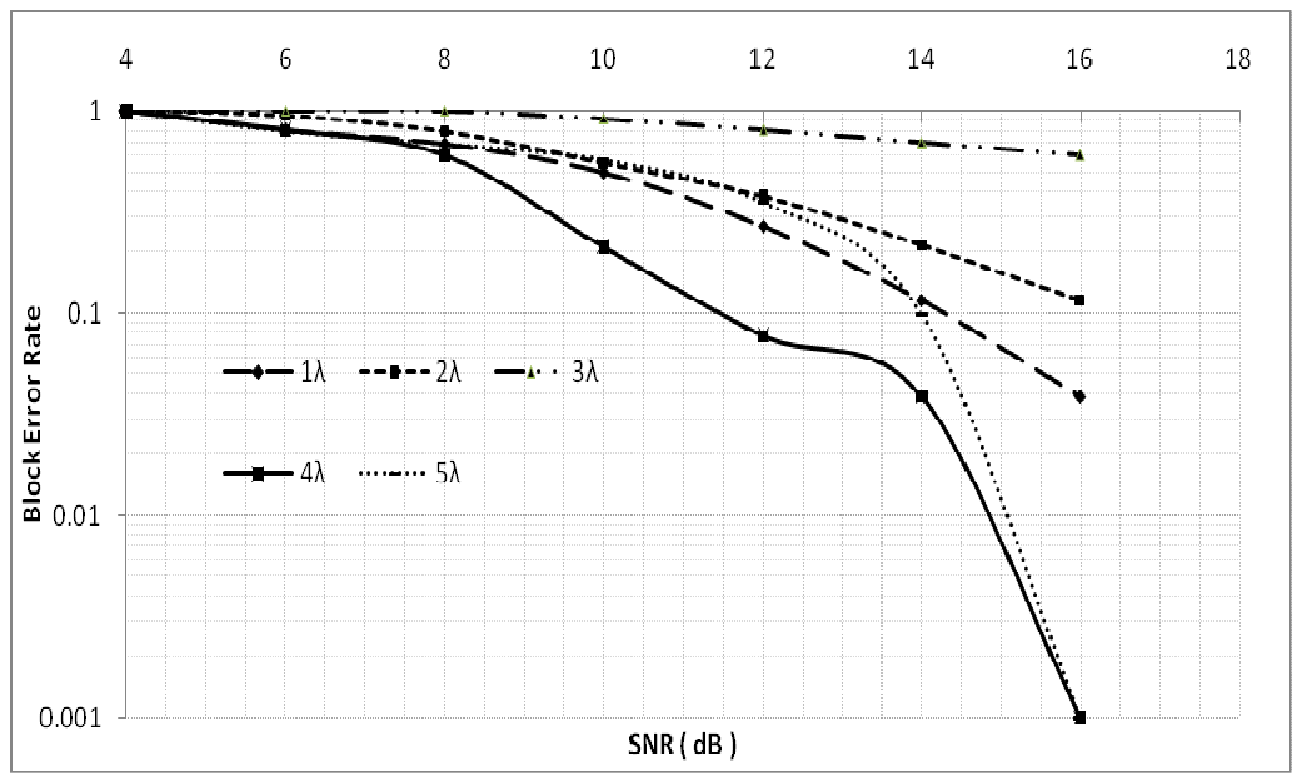

Figure 11. BLER v/s SNR Plot for Open-Loop MIMO 
International Journal of Wireless \& Mobile Networks (IJWMN) Vol. 4, No. 6, December 2012

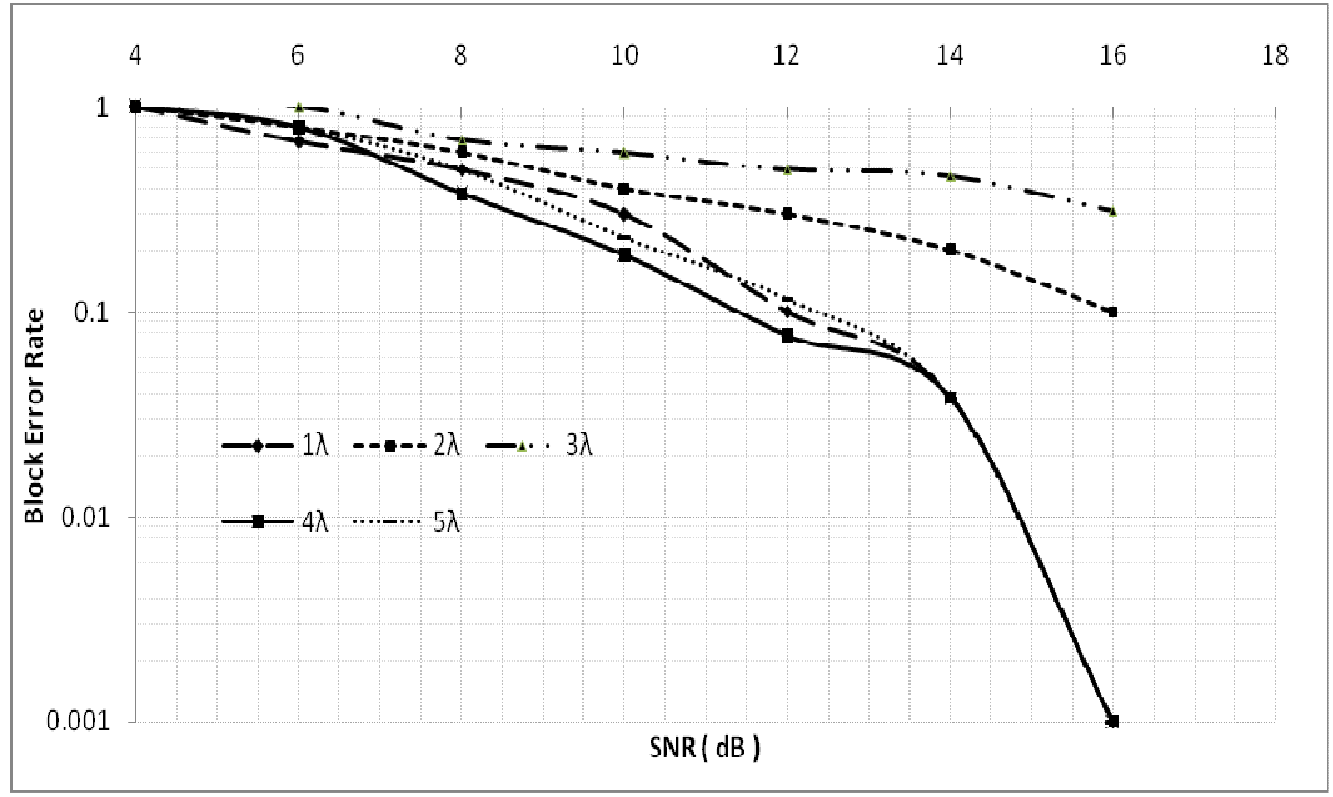

Figure 12. BLER v/s SNR Plot for Close-Loop MIMO

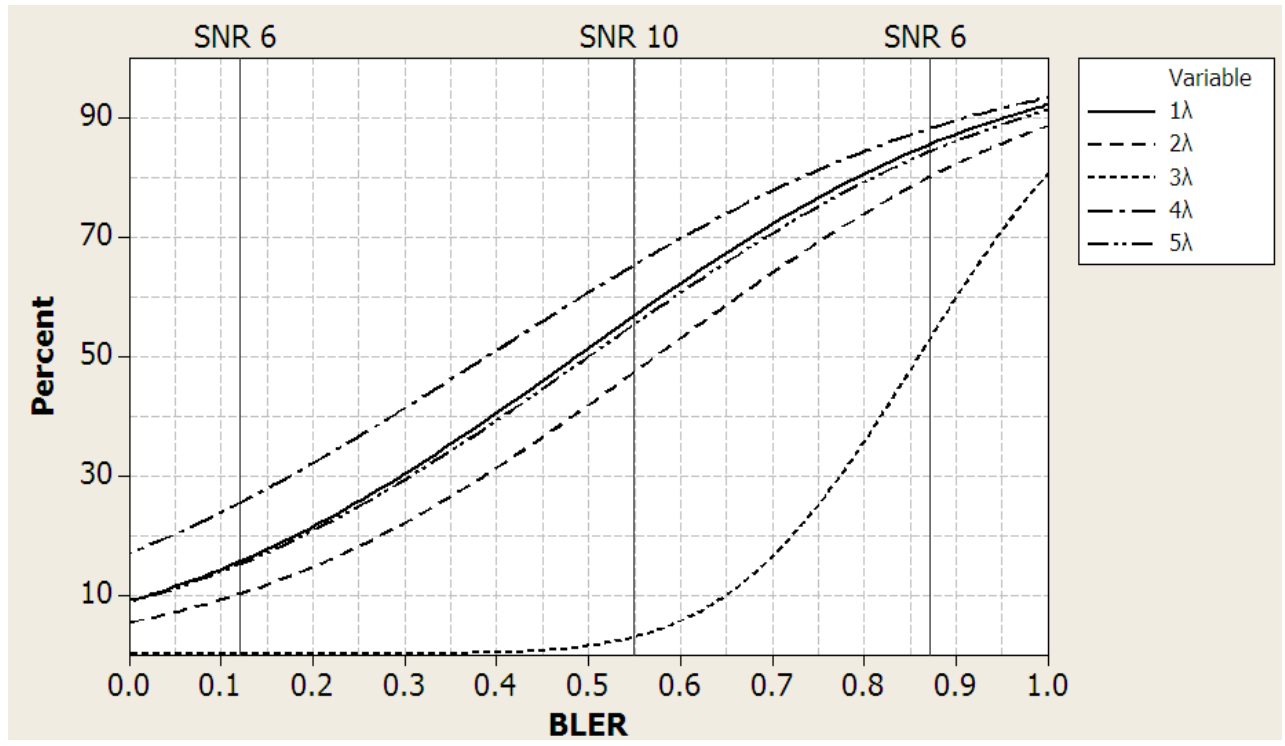

Figure 13. CDF Plot for Open-Loop MIMO 


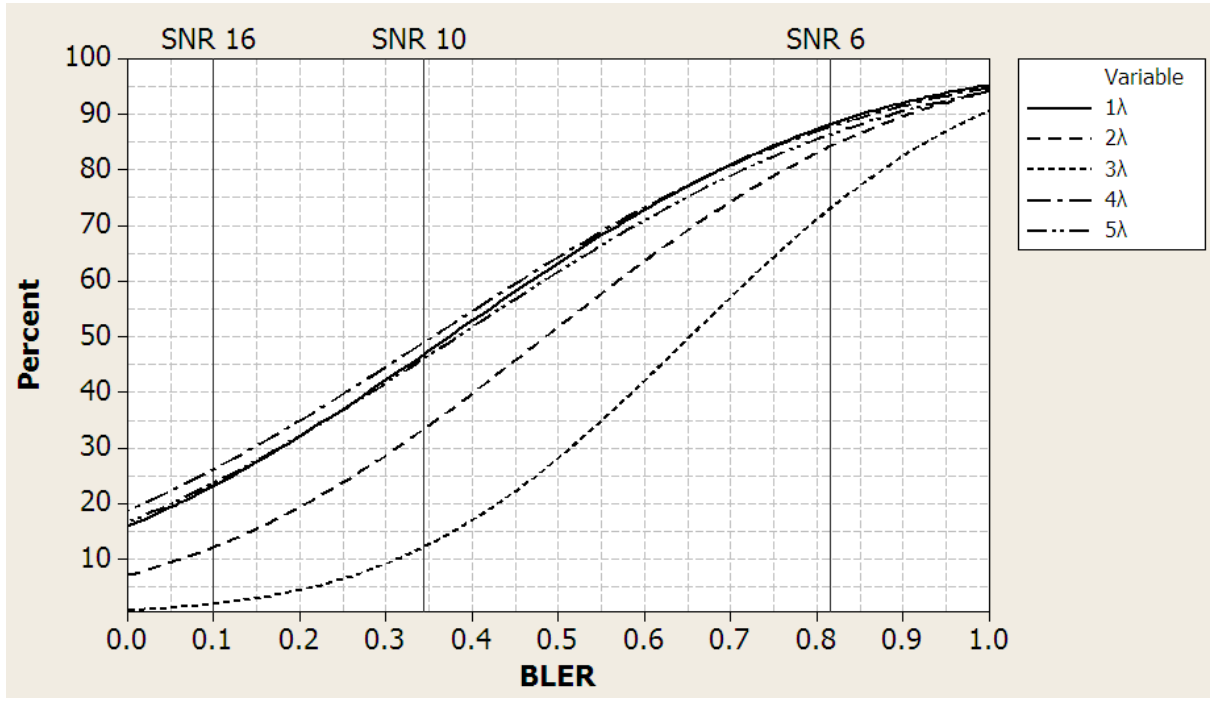

Figure 14. CDF Plot for Open-Loop MIMO

It is observed from figure 11 to 14 that BLER for all condition decreases with increase in SNR. The roll off becomes steeper as SNR ranges beyond $12 \mathrm{~dB}$. For Open loop MIMO small antenna spacing $(<2 \lambda)$ suffers $3 \mathrm{~dB}$ penalty in performance compared to $4 \lambda$ at a reference BLER of $10 \%$, corresponding to BER of 10-2. Remarkably in close loop MIMO the effect of antenna spacing is not significant for a reference of $10 \%$ BLER , as only $0.5 \mathrm{~dB}$ gain is achieved with larger spacing

\section{RESULTS \& DISCUSSIONS}

The effect of BS antenna Spacing on the system performance for open loop and closed loop MIMO is carried out in order to optimize the Base Station Antenna spacing for microcellular Propagation Scenario. The channel model used in the simulation is the Spatial Channel Model Extension (SCME) Urban Micro scenario which is specified in 3GPP [12]. In urban micro-cell scenarios the height of both the antenna at the BS and at the MS is assumed to be well below the tops of surrounding buildings. The streets in the coverage area are classified as 'Main Street', where there is LOS from all locations to the BS, and that intersect the Main Street are referred to as Perpendicular streets. The BS height is set to 10 meter and MS height is set to 1.5 meter with urban microcell length of 500 meters. A three -sector BS antenna and Omni directional MS antenna with $0.5 \lambda$ is used with MIMO set to Spatial Multiplexing (SM) mode, 16-QAM mapping, $1 / 2$ Code rate with subcarrier spacing of $15 \mathrm{KHz}$. The MS speed is kept constant at 20 $\mathrm{Km} / \mathrm{hr}$. The detection is performed with Minimum Mean Square Estimator (MMSE) and a reference Transmit power is $10 \mathrm{dBm}$.

Capacity: The Capacity curves for Open-loop and Closed-loop MIMO depicting the effect of variation in BS antenna spacing are determined and the corresponding CDF curve is plotted as shown in figure 6 and 7 . The effect of BS antenna spacing from $0.5 \lambda$ through $4 \lambda$, at relatively lower SNR $(<=5 \mathrm{~dB})$ indicates a marginal increase in capacity with maximum values observed for $4 \lambda$ spacing for both open and close loop MIMO. However, at moderate SNR (5-10dB) and also at relatively higher SNR $(>10 \mathrm{~dB})$, the effect of antenna spacing is more pronounced leading to spectral efficiency gain of nearly $25 \%$ with $4 \lambda$ spacing. 
International Journal of Wireless \& Mobile Networks (IJWMN) Vol. 4, No. 6, December 2012

It is observed that at lower SNR $(<5 \mathrm{~dB})$, close loop MIMO provides a noticeable improvement of about 3\% (0.027 bits/sec/Hz) over open loop MIMO leading to SNR gain of around $2 \mathrm{~dB}$. The capacity improvement at moderate SNR range is around $1.9 \%(0.049 \mathrm{bits} / \mathrm{sec} / \mathrm{Hz})$ and at higher SNR values $(>10 \mathrm{~dB})$ is $1.7 \%(0.057 \mathrm{bits} / \mathrm{sec} / \mathrm{Hz})$.

Throughput: The CDF of throughput curves for open loop and closed loop MIMO are shown in figures 9 and 10. It is observed that performance degrades in open loop MIMO compared to close loop MIMO for all SNR range. For moderate SNR $(10 \mathrm{~dB})$ the throughput is $14 \mathrm{Mbps}$ for $2 \lambda$ spacing, $17 \mathrm{Mbps}$ for $1 \lambda$ spacing, and $21 \mathrm{Mbps}$ for $4 \lambda$ spacing. The improvement in throughput from open loop to close loop is around $21 \%$ (11.66 Mbps v/s $15.86 \mathrm{Mbps})$. As the channel condition improves ( $>10 \mathrm{~dB}$ ), increase in spacing from $0.5 \lambda$ to $4 \lambda$ has distinct effect on throughput, $4 \lambda$ achieves roughly $3 \mathrm{~dB}$ SNR gain in open loop, and $1.2 \mathrm{~dB}$ SNR gain in close loop over $2 \lambda$ with maximum throughput on the order of 20 Mbps.

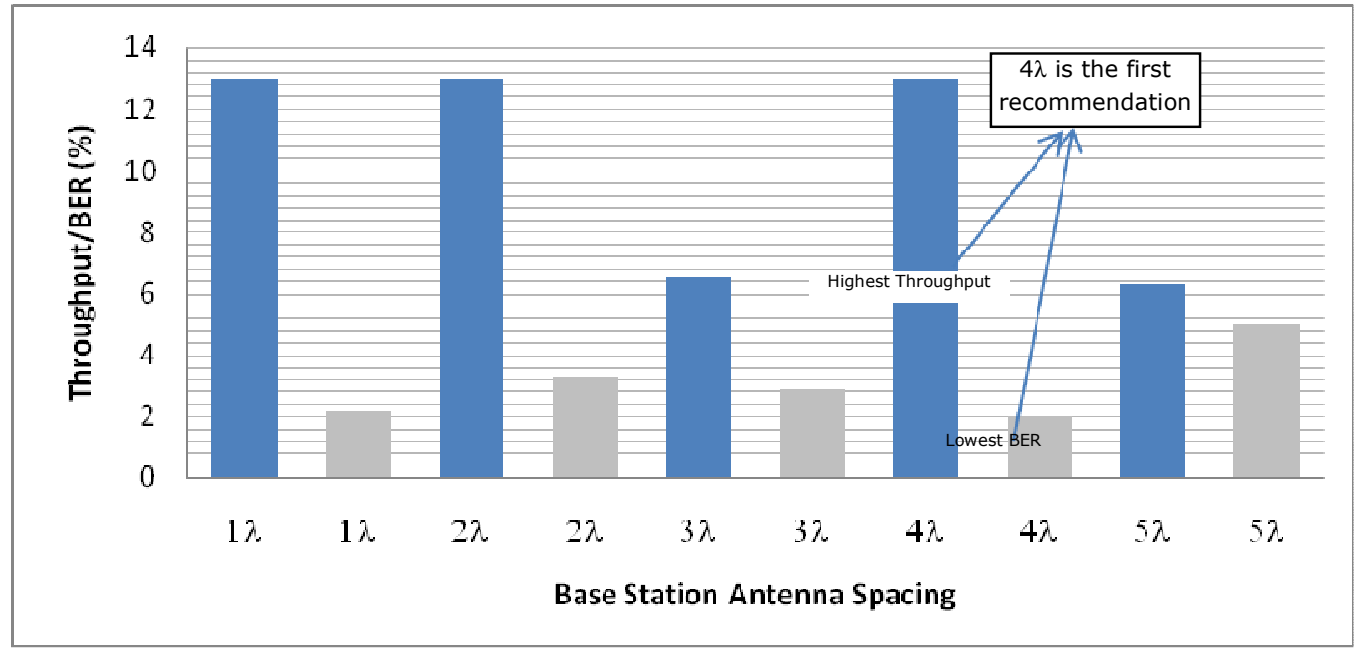

Figure 15: Throughput and BER plots with BS antenna spacing for $2 \times 2$ MIMO (at 2 SNR).

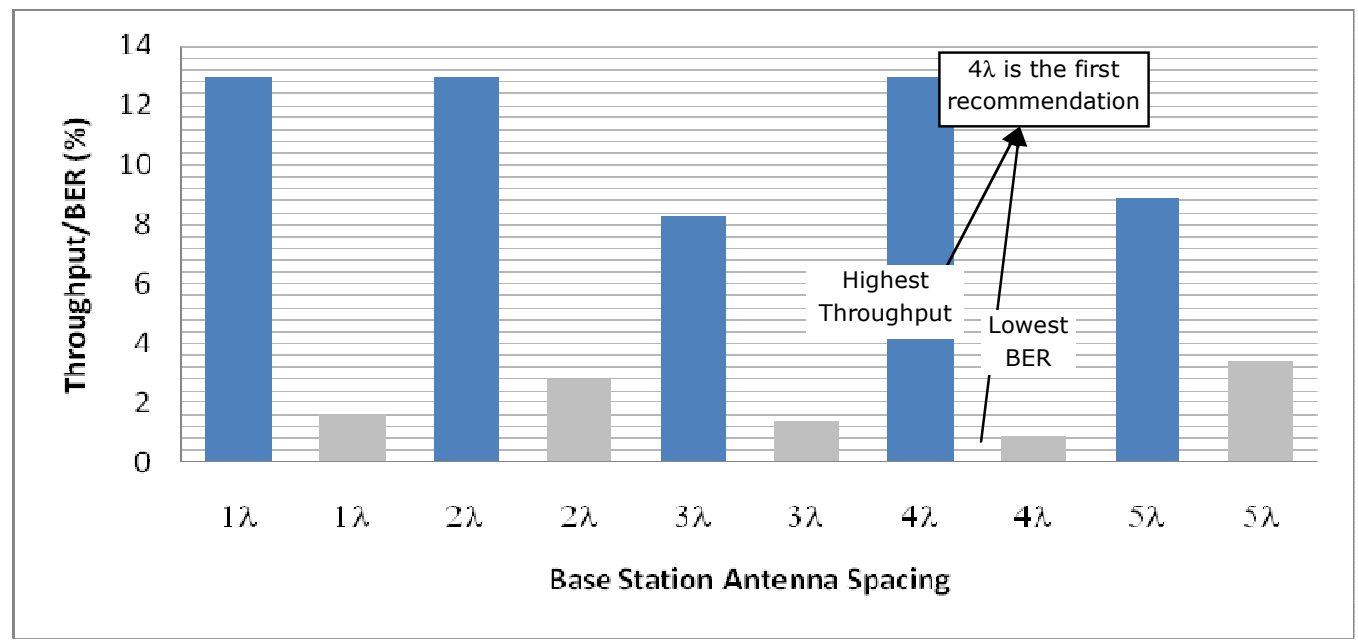

Figure 16: Throughput and BER plots with BS antenna spacing for $2 \times 2$ MIMO (at 8 SNR) 
International Journal of Wireless \& Mobile Networks (IJWMN) Vol. 4, No. 6, December 2012

Bit Error Rate: The CDF of BER curves for Open-loop and Closed-loop MIMO depicting the effect of variation in BS antenna spacing are as shown in figure 13 and 14. It is obvious that BER in general, decreases with increase in SNR. The roll off becomes steeper as SNR ranges beyond $12 \mathrm{~dB}$. In open loop system at a reference BER of $10 \%$, base station spacing less than $2 \lambda$ suffers $4 \mathrm{~dB}$ penalty compared to $4 \lambda$. Remarkably, in close loop MIMO the effect of antenna spacing is not significant for a reference of $10 \%$ BER, as only $1 \mathrm{~dB}$ gain is achieved with larger spacing.

\section{CONCLUSION}

The performance improvement of 2X2 MIMO LTE downlink with increase in base station antenna spacing is quantitatively reported and discussed. The performance improvement is attributed to existence of highly uncorrelated MIMO channels for relatively large antenna spacing. The base station antenna spacing is found to be a key factor in influencing the overall performance of the system. It is observed that capacity is more or less dependent on SNR conditions, however throughput and BER are found to be sensitive towards antenna spacing variations. It is observed that at low SNR $(<5 \mathrm{~dB})$, antenna spacing of $1 \lambda$ is preferred on account of throughput compared to $2 \lambda / 4 \lambda$. BER for both close loop and open loop does not have any significant effect over antenna spacing. With increase in SNR (10 dB), close loop MIMO significantly improves BER, Moreover, $4 \lambda$ spacing shows a distinct effect on throughput with a rise of almost 50-60\% compared to other spacing. At SNR beyond 12dB, large antenna spacing gives almost equal values of throughput in open loop and close loop. However BER is significantly reduced from average value of $10 \%$ to $1 \%$ for $4 \lambda$ in open loop MIMO.

\section{ACKNOWLEDGEMENT}

We thank department of Information Technology (CC\&BT Group), Ministry of Communication and Information Technology, Govt. of India, for there financial support for the research project File No.14(10)/2010-CC\&BT.

\section{REFERENCE}

[1] G. J. Foschini and M. J. Gans, "On limits of wireless communications in a fading environment when using multiple antennas,"Wireless Personal Communications, vol. 6, no. 3, pp.311-335, 1998][2] E. Telatar, "Capacity of multi-antenna Gaussian channels," European Transactions on Telecommunications, vol. 10, no. 6,pp. 585-595, 1999.]

[2] 3GPP TS, "LTE physical layer-general description," 36.201-v1.0.0, http://www.3gpp.org/ftp/Specs/archive/36\%5Fseries/36.201.[4] 3GPP TS, "Physical channels and modulation," http://www.3gpp.org/ftp/Specs/archive/36\%5Fseries/36.211.

[3] D. S. Shiu, G. J. .Foschini, M. J. Gans and J. M. Kahn, "Fading Correlation and its Effects on the Capacity of Multielement Antenna Systems,” IEEE Trans. on Comm., vol. 48, no. 3, pp. 502 - 512 , Mar. 2000.

[4] J. Zhang, H. Zhang, N. V. Wales, A. Reid and V. Stolpman, "Closed-Loop MIMO Precoding with Limited Feedback," IEEE 802.16 Broadband Wireless Access Working Group, http://ieee802.org/16, Aug. 2004.]

[5] Carsten F. Ball1, Robert Müllner1, Johann Lienhart2 and Hubert Winkler2 Carsten F. Ball1, Robert Müllner1, Johann Lienhart2 and Hubert Winkler]. 
International Journal of Wireless \& Mobile Networks (IJWMN) Vol. 4, No. 6, December 2012

[6] Sebastian Caban1, Jos_e A. Garc_a-Naya2, Christian Mehlf $\square$ uhrer1, Luis ," Measuring the Closed-Loop Throughput of 2x2 HSDPA over TX Power and TX Antenna Spacing”

[7] Zhao, X., Kivinen, J., Vainikainen, P., Skog, K.. Propagation characteristics for wideband outdoor mobile communications at $5.3 \mathrm{GHz}$. IEEE Journal on Selected Areas in Communications 2002;20(3):507\{514. doi: 10.1109/49.995509.

[8] Jungnickel, V., Pohl, V., von Helmolt, C.. Capacity of MIMO systems with closely spaced antennas. IEEE Communications Letters 2003;7(8):361 \{363. doi: 10.1109/LCOMM.2003.815644

[9] Thomas, T.A., Desai, V., Kepler, J.F.. Experimental MIMO comparisons of a 4-element uniform linear array to an array of two cross polarized antennas at $3.5 \mathrm{GHz}$. In: Proc. of VTC 2009 Fall. 2009:

[10] Chizhik, D., Rashid-Farrokhi, F., Ling, J., Lozano, A.. E_ect of antenna separation on the capacity of BLAST in correlated channels. IEEE Communications Letters 2000;4(11):337\{339. doi: $10.1109 / 4234.892194$

[11] MIMO Technologies in 3GPP LTE and LTE-Advanced Juho Lee,1 Jin-Kyu Han,1 and Jianzhong (Charlie) Zhang2 Hindawi Publishing Corporation EURASIP Journal on Wireless Communications and Networking Volume 2009, Article ID 302092, 10 pages doi:10.1155/2009/302092]

[12] WINNER II Channel Models, D1.1.2 V1.2, IST-4-027756 WINNER II Deliverable, Feb. 4th 2008.

[13] Mir Ghoraishi, Jun-ichi Takada, Tetsuro Imai , "Microcell Urban Propagation Channel Analysis Using Measurement Data" 0-7803-9152-7/05/\$20.00 (c) 2005 IEEE

[14] Lizhong Zheng, Member, IEEE, and David N. C. Tse, Member, IEEE, "Diversity and Multiplexing: A Fundamental Tradeoff in Multiple-Antenna Channels," IEEE Transactions on Information Theory, Vol. 49, No. 5, MAY 2003

[15] R. Bhagavatula, A. Forenza, and R. W. Heath, Jr., "Impact of Antenna Array Configurations on Adaptive Switching in MIMO Channels," Proc. of Int. Symp. on Wireless Pers. Mult. Comm., Sept. 2006.

[16] Angel Lozano, Senior Member, IEEE, and Nihar Jindal, Member, "Macro-Cellular Capacity Enhancement using Spatial Multiplexing MIMO with Linear Antenna Arrays" IEEE IEEE TRANSACTIONS ON WIRELESS COMMUNICATIONS, VOL. 9, NO. 1, JANUARY 2010

[17] L. Zheng and D. N. C. Tse, "Diversity and multiplexing: A fundamental tradeoff in multiple antenna channels," IEEE Trans. Inf. Theory, vol. 49, pp. 1073-1096, May 2003.

[18] Pedro Vieira, Paula Queluz and Ant'onio Rodrigues, Macro-Cellular Capacity Enhancement using Spatial Multiplexing MIMO with Linear Antenna Arrays.

\footnotetext{
Authors

Dr. Sunil Joshi was born in Jodhpur, India in 1967. He received B.E. (Hons) in 1990 in Electronics \& Communication Engineering and M.E. in Year 2000 in Digital Communication from MBM Engineering College, JNV University, Jodhpur (India). He taught at various capacities in Diploma and Degree level technical Institutions. He did his Ph.D. in Year 2006 in "Propagation Studies of Millimeter Waves" from Microwave Systems Group Departmentof Electronics \& Communication Engineering Mailviya National Institute of Technology, University of Rajasthan, Jaipur. Presently he is Head of Electronics \& Communication Department in the College of Technology \& Engineering, Maharana Pratap University of Agriculture \& Technology, Udaipur.
}

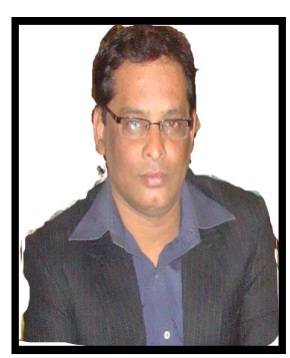


International Journal of Wireless \& Mobile Networks (IJWMN) Vol. 4, No. 6, December 2012

Deepak Gupta was born in Jodhpur, India in 1973. He received B.E. (Hons) in 1995 in Electronics \& Communication Engineering and M.E. (Gold Medallist) in Digital Communication from MBM Engineering College, JNV University, Jodhpur (India). He joined as lecturer in Electronics in Vidya Bhawan Polytechnic College, Udaipur in 1996; presently he is Head of Electronics Department in the Institute. He is currently pursing Ph.D. in "Channel Optimization Techniques in Urban Outdoor and Indoor Indian Environment using Multiple Input Multiple-Output Technology".

S.Z. Haque He is a Scientist F, Director \& HOD (Broadband Technologies) Member Secretary, Working Group Department of Electronics \& Information Technology, Ministry of Communications \& Information Technology ,Government of India.Electronics Niketan, 6 CGO Complex, New Delhi

Neha Kothari She did her B.E. in Electronics and communication engineering from Geetajnali Institute of Technical Studies, Dabok, Udaipur. She is research Scholar in Electronics \& Communication Engineering Department in College of Technology \& Engineering, Maharana Pratap University of Agriculture \& Technology, Udaipur and is working on department of Information Technology (CC\&BT Group), Ministry of Communication and Information Technology, Govt. of India project "Channel/ Cluster Optimization In Wireless Broadband Access Using MIMO and Multiple Antenna Techniques."

P.C. Bapna was born in Udaipur, India in 1956. He did his engineering studies from University of Engineering, Mysore and Complete his MBA Degree from Mohan Lal Sukhadiya University, Udaipur (Rajasthan). Presently he is Associate Professor in Electronics \& Communication Engineering Department in College of Technology \& Engineering, Maharana Pratap University of Agriculture \& Technology, Udaipur. He is currently pursing
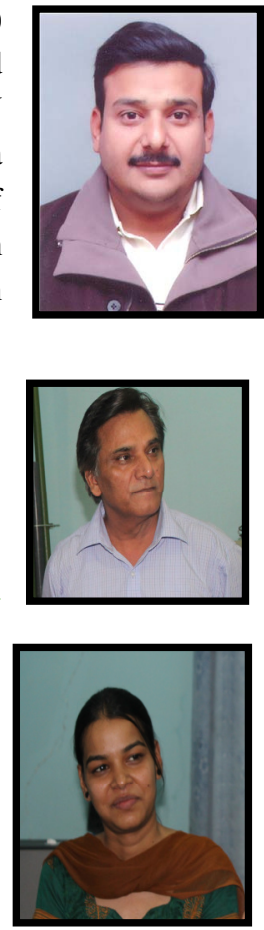
Ph.D. studies. 